\title{
Integrable Quantum Field Theories with Unstable Particles
}

\author{
J. Luis Miramontes* \\ Departamento de Física de Partículas, \\ Facultad de Física \\ Universidad de Santiago de Compostela \\ E-15706 Santiago de Compostela, Spain \\ E-mail: 'miramont@fpaxp1.usc.es'
}

ABSTRACT: The structure of a new family of factorised $S$-matrix theories with resonance poles is reviewed. They are conjectured to correspond to the Homogeneous sine-Gordon theories associated with simply laced compact Lie groups. Two of their more remarkable properties are, first, that some of the resonance poles can be traced to the presence of unstable particles in the spectrum, and, second, that they involve several independent mass scales. The conjectured relationship with the simply laced HSG theories has been checked by means of the Thermodynamic Bethe ansatz (TBA) and, more recently, through the explicit calculation of the Form Factors. The main results of the TBA analysis are summarized.

The construction of solvable models that capture realistic properties of quantum particles is one of the classical motivations for the study of two-dimensional integrable quantum field theories. However, despite the fact that almost all known particles are unstable "iin, the vast majority of the integrable models considered so far lack the feature of including unstable particles. The aim of this talk is to present a new family of factorised $S$-matrix theories [2] conjectured to provide the exact solution for the Homogeneous

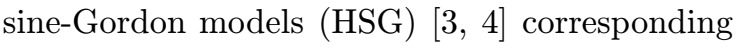
to simply-laced Lie groups. The semi-classical spectrum of these models consists of a finite number of solitonic particles attached to the positive roots of a Lie algebra, but only those associated with the simple roots turn out to correspond to stable particles in the quantum theory.

Although it is very difficult to distinguish physically between an unstable particle of long lifetime and a stable particle (e.g., the proton), axiomatic $S$-matrix theory makes a big distinc-

\footnotetext{
*Based on work done in collaboration with O.A. Castro-Alvaredo, C.R. Fernández-Pousa, A. Fring and C. Korff.
}

tion between them. The reason is that it is based on asymptotic states that exist for arbitrarily long time and, hence, can only contain stable particles. In contrast, the basic property of a long-lived unstable particle, and the one by means of which it is usually observed, it that it corresponds to a 'resonance' in interactions among the stable particles. Therefore, if two stable particles scatter at centre-of-mass energy $\sqrt{s}$ close to the mass of an unstable state with appropriate quantum numbers, the corresponding $S$-matrix amplitude is expected to exhibit a complex pole at $s_{R}=(M-i \Gamma / 2)^{2}$ in the second Riemann sheet. The position of the pole is given by the mass, $M$, and decay width, $\Gamma$, of the resonance, whose inverse is the lifetime of the unstable particle: $\tau=\hbar / \Gamma$. If the lifetime is long or, equivalently, if $\Gamma \ll M$, the form of the pole is given by the Breit-Wigner resonance formula [i]

$$
S \simeq 1-i \frac{2 M \Gamma}{s-M^{2}+i M \Gamma} .
$$

In a generic theory, unstable particles can also induce other singularities like complex thresholds and cuts. The simplest would correspond to the 
production of the unstable particle together with one stable particle. However, since the unstable particle decays, this kind of process would lead to particle production and, hence, it has to be absent if the theory is integrable. Therefore, we will assume that resonance poles are the only trace of unstable particles in an integrable quantum field theory.

There is a different HSG theory for each choice of a compact simple Lie group $G$ (with Lie algebra $g$ ) and a positive integer $k$. It describes an integrable massive perturbation of the conformal field theory (CFT) associated with the coset $G_{k} / U(1)^{\times r_{g}}$, where $r_{g}=\operatorname{rank} G$, or, equivalently, of the theory $G$-parafermions at level$k[\overline{4}]$. When $G$ is simply laced, the central charge of the CFT and the conformal dimension of the perturbation are given by

$$
\begin{aligned}
& c_{\mathrm{CFT}}=\frac{k \operatorname{dim} G}{k+h_{g}}-r_{g}=\frac{k-1}{k+h_{g}} h_{g} r_{g}, \\
& \Delta=\bar{\Delta}=\frac{h_{g}}{k+h_{g}}<1,
\end{aligned}
$$

where $h_{g}$ is the Coxeter number of $G$. The defining action of the HSG models is

$$
\begin{aligned}
S_{\mathrm{HSG}} & =\frac{1}{\beta^{2}}\left\{S_{\mathrm{GWZW}}[h]\right. \\
+ & \left.\frac{m^{2}}{\pi} \int d^{2} x\left\langle\Lambda_{+}, h^{\dagger} \Lambda_{-} h\right\rangle\right\},
\end{aligned}
$$

whose equations of motion are non-abelian affine Toda equations [3. 3 ]. In $S_{\mathrm{HSG}}, h=h(t, x)$ is a bosonic field taking values in $G$ and $S_{\mathrm{GWZW}}[h]$ is the gauged Wess-Zumino-Witten action corresponding to the coset $G_{k} / U(1)^{\times r_{g}}$. The parameters $m$ and $\beta^{2}=1 / k+O\left(1 / k^{2}\right)$ are the bare mass scale and the coupling constant, whose quantization is required in order to make sense of the WZW term. $\Lambda_{+}$and $\Lambda_{-}=i \overrightarrow{\lambda_{ \pm}} \cdot \vec{H}$, are two arbitrary constant elements in the fundamental Weyl chamber of the Cartan subalgebra of $g$ associated with the maximal torus $U(1)^{\times r_{g}}$. The HSG theories are quantum integrable for any choice of $G$ and for any value of $\Lambda_{ \pm}[\overline{4}]_{-1}^{-1}$, which, in the quantum theory, imply the existence of $2 r_{g}-1$ different mass scales. The HSG theories are not parity invariant unless $\Lambda_{+}$and $\Lambda_{-}$are chosen to be parallel.
The semiclassical spectrum of the $G_{k}-\mathrm{HSG}$ theory consists of towers of $k-1$ soliton particles attached to each positive root $\vec{\alpha}$ of $g$ with masses [ín]

$$
M_{\vec{\alpha}}[n]=\frac{k}{\pi} m_{\vec{\alpha}} \sin \left(\frac{\pi n}{k}\right), \quad n=1, \ldots, k-1 .
$$

For a fixed root $\vec{\alpha}$, this spectrum is identical to that one of the minimal $A_{k-1} S$-matrix theory, which corresponds to the $S U(2)_{k}-\mathrm{HSG}$ model. However, the overall mass scale

$$
m_{\vec{\alpha}}=2 m \sqrt{\left(\vec{\alpha} \cdot \vec{\lambda}_{+}\right)\left(\vec{\alpha} \cdot \vec{\lambda}_{-}\right)}
$$

is different for each tower of particles. Consider now the unique decomposition of a positive root as a linear combination of simple roots: $\vec{\alpha}=$ $\sum_{i=1}^{r_{g}} p_{i} \vec{\alpha}_{i}$. Then, eq. (产充) satisfies

$$
M_{\vec{\alpha}}[n] \geq \sum_{i=1}^{r_{g}} M_{\vec{\alpha}_{i}}\left(n p_{i}\right)
$$

which indicates that the soliton particle $(\vec{\alpha}, n)$ is unstable and decays into particles associated with the simple roots [i2i]. Moreover, for any three $\operatorname{roots} \vec{\alpha}, \vec{\beta}$, and $\vec{\alpha}+\vec{\beta}$ of $g$, it can be easily checked that

$$
m_{\vec{\alpha}+\vec{\beta}}^{2}=m_{\vec{\alpha}}^{2}+m_{\vec{\beta}}^{2}+2 m_{\vec{\alpha}} m_{\vec{\beta}} \cosh \sigma_{\vec{\alpha}, \vec{\beta}},
$$

where

$$
\sigma_{\vec{\alpha}, \vec{\beta}}=\frac{1}{2} \ln \frac{\left(\vec{\alpha} \cdot \vec{\lambda}_{+}\right)\left(\vec{\beta} \cdot \vec{\lambda}_{-}\right)}{\left(\vec{\alpha} \cdot \vec{\lambda}_{-}\right)\left(\vec{\beta} \cdot \vec{\lambda}_{+}\right)} .
$$

These equations, together with $(\underline{\underline{\underline{2}}})$ ), establish the relationship between the arbitrary constants $\vec{\lambda}_{ \pm}$ and the different scales that determine the semiclassical mass spectrum of stable and unstable particles.

In [in], it was conjectured that the exact solution of the simply laced HSG theories is provided by a diagonal $S$-matrix. In this solution, the exact spectrum of stable particles coincides with the semi-classical spectrum of soliton particles of the HSG theory associated with the simple roots of the algebra, and there is an independent mass scale attached to each simple root or, equivalently, to each node of the Dynkin diagram of $g: m_{1}, \ldots, m_{r_{g}}$. Following [i7n, particles will be labelled by two quantum numbers $(a, i)$, with 
$1 \leq a \leq k-1$ and $1 \leq i \leq r_{g}$, and $S_{a b}^{i j}(\theta)$ will be the two-particle scattering amplitude corresponding to the process where the particle $(a, i)$ initially is on the left-hand-side of the particle $(b, j)$. For particles associated to the same simple root, $i=j$, the amplitude is provided by the minimal $S$-matrix associated to $A_{k-1}$

$$
\begin{aligned}
S_{a b}^{i i}(\theta) & =S_{a b}^{A_{k-1}}(\theta)=(a+b)_{\theta}(|a-b|)_{\theta} \\
& \times \prod_{n=1}^{\min (a, b)-1}(a+b-2 n)_{\theta}^{2},
\end{aligned}
$$

where we have introduced the block notation $(x)_{\theta}=$ $\sinh \frac{1}{2}\left(\theta+i \frac{\pi x}{k}\right) / \sinh \frac{1}{2}\left(\theta-i \frac{\pi x}{k}\right)$. On the other hand, the scattering between solitons associated to different simple roots is described by

$$
\begin{aligned}
S_{a b}^{i j}(\theta) & =\left(\eta_{i, j}\right)^{a b} \prod_{n=0}^{\min (a, b)-1}(-|a-b|-1-2 n)_{\theta+\sigma_{i j}} \\
& \neq S_{b a}^{j i}(\theta)
\end{aligned}
$$

if $\vec{\alpha}_{i}+\vec{\alpha}_{j}$ is a root of $g$, and by $S_{a b}^{i j}(\theta)=1$ otherwise. In this equation $\sigma_{i j}=-\sigma_{j i}\left(\sigma_{i i}=0\right)$ are $r_{g}-1$ free real parameters attached to the links of the Dynkin diagram of $g$. They determine the position of the resonance poles or, equivalently, the mass scales of the spectrum of unstable particles. In particular, $S_{a a}^{i j}(\theta)$ has a resonance pole at $\theta=\sigma_{j i}-i \pi / k$ which, for $\sigma_{j i}>0$, should correspond to the unstable soliton particle with mass $M_{\vec{\alpha}_{i}+\vec{\alpha}_{j}}[a]$ in the semiclassical $k \gg h_{g}$ limit. The existence of the $2 r_{g}-1$ free parameters $m_{i}$ and $\sigma_{i j}$ is a consequence of the freedom

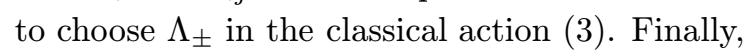
$\eta_{i, j}=\eta_{j, i}^{*}$ for $i \neq j\left(\eta_{i i}=1\right)$ are arbitrary $k$ th roots of -1 whose presence is required to satisfy both the crossing relations and the bootstrap equations [2].

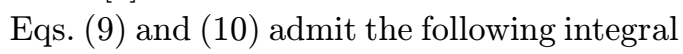
representation [i]in]

$$
\begin{aligned}
& S_{a b}^{i j}(\theta)=\sqrt{\eta_{i, j}^{-2 k \mathcal{C}_{a b}^{-1}}} \exp \int \frac{d t}{t} \mathrm{e}^{-i t\left(\theta+\sigma_{i j}\right)} \\
& \times\left(2 \cosh \frac{\pi t}{k}-\mathcal{I}^{(g)}\right)_{i j}\left(2 \cosh \frac{\pi t}{k}-\mathcal{I}\right)_{a b}^{-1},
\end{aligned}
$$

where $\mathcal{C}=2-\mathcal{I}$ and $\mathcal{C}^{(g)}=2-\mathcal{I}^{(g)}$ are the Cartan matrices of $A_{k-1}$ and $g$, respectively. The scattering matrices of the simply laced HSG theories have been recently generalized in a Lie algebraic sense in [8], where a new factorised $S$-matrix theory is associated to each pair $(\widetilde{g} \mid g)$ of simply laced Lie algebras. In this construction, the $G_{k}-\mathrm{HSG}$ $S$-matrix corresponds to the pair $\left(A_{k-1} \mid g\right)$.

So far, the relationship between the proposed $S$-matrices and the simply laced HSG theories has been checked by means of the Thermodynamic Bethe ansatz (TBA) [7.7] and, more recently, through the explicit calculation of the Form Factors $[9.9$. Assuming that all particles are of fermionic type $\left(S_{a a}^{i i}(0)=-1\right)$, the TBA equations read

$$
\epsilon_{a}^{i}(\theta)+\sum_{b, j} \Phi_{a b}^{i j} * L_{b}^{j}(\theta)=R M_{a}^{i} \cosh \theta,
$$

where $M_{a}^{i}=m_{i} \sinh (\pi a / k)$ and $L_{b}^{j}(\theta)=\ln (1+$ $\left.\exp -\epsilon_{b}^{j}(\theta)\right)$. Recall that the scattering amplitudes given by eqs. $\left(\overline{\bar{g}_{1}^{\prime}}\right)-\left(\begin{array}{l}1 \\ 1\end{array} \overline{1}_{1}^{\prime}\right)$ are not parity symmetric. Actually, it is easy to check that they satisfy the Hermitian analyticity condition $S_{a b}^{i j}(\theta)=$ $\left[S_{b a}^{j i}\left(-\theta^{*}\right)\right]^{*} \neq S_{a b}^{i j}(\theta)$ which, for real values of the rapidity, implies that

$$
\Phi_{a b}^{i j}(\theta)=-i \frac{d}{d \theta} \ln S_{a b}^{i j}(\theta)=\Phi_{b a}^{j i}(-\theta) \neq \Phi_{a b}^{i j}(-\theta)
$$

and, hence, $\epsilon_{a}^{i}(\theta) \neq \epsilon_{a}^{i}(-\theta)$. Consequently, the finite size scaling function is given by

$$
c(R)=\frac{3 R}{\pi^{2}} \sum_{a, i} M_{a}^{i} \int_{0}^{\infty} d \theta \cosh \theta\left(L_{a}^{i}(\theta)+L_{a}^{i}(-\theta)\right) .
$$

An important quantity that is provided by the TBA analysis is the value of $c(R)$ in the $R \rightarrow 0$ limit, which corresponds to the the central charge of the CFT that governs the ultra-violet (UV) limit of the $S$-matrix theory, assuming that it is unitary. Taking into account that the kernels $\Phi_{a b}^{i j}\left(\theta-\sigma_{i j}\right)$ are strongly peaked at $\theta=0$,

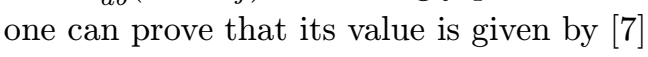

$$
\lim _{R \rightarrow 0} c(R)=\frac{6}{\pi^{2}} \sum_{a, i} \mathcal{L}\left(f_{a}^{i}\right)
$$

where $\mathcal{L}(x)$ is Roger's dilogarithm function, and $f_{a}^{i}=\left(1+\exp \epsilon_{a}^{i}(0)\right)^{-1}$, for $a=1, \ldots, k-1$, and $i=1, \ldots, r_{g}$, satisfy the constant TBA equations

$$
\sum_{b=1}^{k-1} \mathcal{C}_{a b} \ln f_{b}^{i}=\sum_{j=1}^{r_{g}} \mathcal{C}_{i j}^{(g)} \ln \left(1-f_{a}^{j}\right) .
$$


These equations were considered before in the context of the 'restricted solid-on-solid' models [1 [10'] and solved in terms of Weyl characters, with the result that the value of $c(R)$ for $R \rightarrow 0$ indeed coincides with the central charge of the CFT associated with the coset $G_{k} / U(1)^{r_{g}}$ for any $G$ and

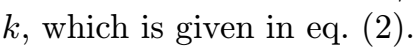

All this confirms that the $S$-matrices defined by eqs. $(\overline{9} \cdot \overline{9})-\left(1 \overline{1} \overline{1}_{1}^{\prime}\right)$ describe massive integrable perturbations of the theory $G$-parafermions at level$k$ for any (finite) value of the $2 r_{g}-1$ free parameters $\left(m_{i}, \sigma_{j l}\right)$, which strongly supports the conjectured connection with the HSG models. Additional support is provided by the recent application of the Form Factor program to the $\mathrm{SU}(3)_{2}-$

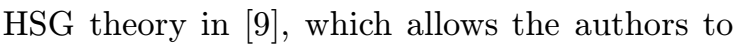
calculate not only the value of the UV central charge $(6 / 5)$ using the Zamolodchikov's $c$-theorem, but also the conformal dimension of various operators including the dimension of the perturbing operator $(3 / 5)$.

Nevertheless, this is not the only information that can be obtained from the TBA analysis. For finite values of $R$, the finite size scaling function $c(R)$ shows a rather characteristic 'staircase pattern' where the number of steps is finite and their position is fixed by the value of the different free parameters entering the definition of the $S$-matrix. This suggests the interpretation of the staircase pattern as a consequence of the change in the number of effective light degrees of freedom produced by the decoupling of the heavy particles, both stable and unstable, compared to the the scale given by the temperature $T \simeq 1 / R$. This provides a strong argument in favour of the interpretation of the resonance poles as a trace of the presence of unstable particles in the spectrum, in agreement again with the expected properties of the HSG models. It is worth noticing that the original staircase models [1] do not allow such a direct physical interpretation for the observed staircase pattern.

Although $c(R)$ has to be calculated numerically, it is possible to understand analytically the physical origin of the staircase behaviour. It will be convenient to separate the TBA kernel in two different parts:

$$
\Phi_{a b}^{i j}(\theta)= \begin{cases}\phi_{a b}(\theta) & ; i=j, \\ \mathcal{I}_{i j}^{(g)} \psi_{a b}\left(\theta+\sigma_{i j}\right) & ; i \neq j,\end{cases}
$$

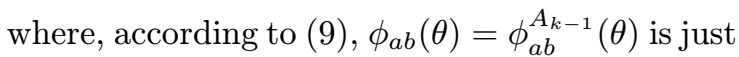
the TBA kernel of the minimal $S$-matrix associated to $A_{k-1}$. Then, the TBA equations (112i) become

$$
\begin{gathered}
\epsilon_{a}^{i}(\theta)+\sum_{b=1}^{k-1}\left[\phi_{a b} * L_{b}^{i}(\theta)+\sum_{j \neq i} \mathcal{I}_{i j}^{(g)} \psi_{a b} * L_{b}^{j}\left(\theta+\sigma_{i j}\right)\right] \\
=R M_{a}^{i} \cosh \theta
\end{gathered}
$$

which shows that whenever the term involving the kernel $\psi_{a b}$ is negligible we are left with the TBA equation for the minimal $A_{k-1} S$-matrix theory: $L_{a}^{i}(\theta) \simeq L_{a}^{\min }(\theta)$. Moreover, standard arguments show that $L_{a}^{i}(\theta) \simeq 0$ if $m_{i} \gg 2 / R$ due to the dominance of the energy term, which allows one to show that the effect of $\psi_{a b}$ is negligible for

$$
\left|\sigma_{i j}\right| \gg-\ln \left(R^{2} m_{i} m_{j} / 4\right) .
$$

Recall that $S_{a b}^{i j}(\theta)$ exhibits complex poles whose position is of the form $\theta=\sigma_{j i}-i \pi n / k$, where $n$ is some positive integer. For $\sigma_{j i} \gg 0$ and $n \ll k$, corresponding to $\Gamma \ll M$ in $\left(\begin{array}{l}1 \\ 1 \\ 1\end{array}\right)$ (1) they are conjectured to indicate the presence of unstable particles whose mass scale is given by

$$
\begin{aligned}
m_{R_{i j}}^{2} & =m_{i}^{2}+m_{j}^{2}+2 m_{i} m_{j} \cosh \left|\sigma_{i j}\right| \\
& \simeq m_{i} m_{j} \mathrm{e}^{\left|\sigma_{i j}\right|} .
\end{aligned}
$$

Thus, the condition to neglect the term involving $\psi_{a b}$ in ( (1) $\left.\overline{1}^{\prime}\right)$ is just $m_{R_{i j}} \gg 2 / R$ which means that the unstable particles related to $\left|\sigma_{i j}\right|$ are heavy and, hence, they are decoupled from the effective theory at the scale fixed by $R$. Notice that the previously mentioned result that $L_{a}^{i}(\theta) \simeq 0$ if $m_{i} \gg 2 / R$ admits a similar interpretation by changing unstable particles by stable ones. Tak-

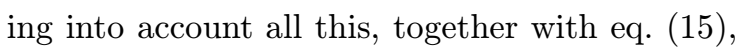
we can sketch the following conjecture about the staircase behaviour of the scaling function:

1) $c(R) \simeq 0 \quad$ if

$$
\frac{2}{R} \ll m_{i_{1}}, \ldots, m_{i_{r_{g}}}, m_{R_{i j}} \quad \text { (deep IR) }
$$


2) $c(R) \simeq n \frac{2(k-1)}{k+2} \quad$ if $m_{i_{1}}, \ldots, m_{i_{n}} \leq \frac{2}{R} \ll m_{i_{n+1}}, \ldots, m_{i_{r_{g}}}, m_{R_{i j}}$, for $n=1, \ldots, r_{g}$,

3) $c(R) \simeq \frac{k-1}{k+h_{g}} h_{g} r_{g} \quad$ if $m_{i_{1}}, \ldots, m_{i_{r_{g}}}, m_{R_{i j}} \ll \frac{2}{R} \quad($ deep UV $)$, and always for all $i \neq j$.

Nevertheless, notice that these qualitative arguments do not explain the behaviour of $c(R)$ in the wole range $0 \leq R<\infty$ and, hence, do not allow one to predict the precise number of steps.

- - - In $[\overline{\overline{1}} \bar{T}$, , the-scaling function- $G(R)$-was-numeri' eally-eateutated-fer-the-S $U(-3-)_{k}$-HSG-nedels with$k=2,3,4$ and the results are in agreement with

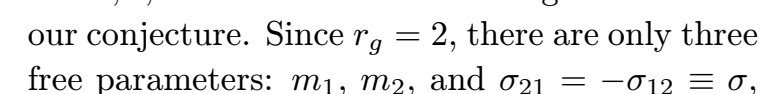
- which-means- that- there is only- one mrass -seale- for-the-unstable particles. - The maximum numrber of steps turns out to be three in this case, which corresponds to the situation when $m_{1} \ll$ $m_{2} \ll m_{R_{12}}$. In contrast, if $m_{1} \simeq m_{2}$ and $\sigma \simeq 0$ there are no steps at all. A remarkable result is that, whenever the unstable particles are much heavier than the stable ones, $m_{R_{12}} \gg m_{1}, m_{2}$, the UV limit of the $S U(3)_{k}-\mathrm{HSG}$ models may be viewed alternatively as a massless $\mathrm{IR} \rightarrow \mathrm{UV}$ flow between two different coset conformal field theories:

$$
\frac{S U(2)_{k}}{U(1)} \times \frac{S U(2)_{k}}{U(1)} \longrightarrow \frac{S U(3)_{k}}{U(1)^{2}}
$$

As a particular case, the flow between the tricritical Ising and the critical Ising model is recovered as a subsystem for $k=2$.

In conclusion, the results obtained so far from

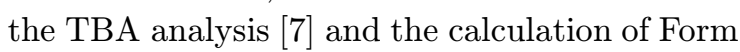
Factors [9] confirm the conjectured relationship between the $S$-matrix theories proposed in [2] and the simply laced HSG theories. Further support could be obtained by extending the explicit calculations to other Lie groups different from $S U(3)$. Moreover, it would be extremely interesting to investigate directly the connection of , the -resonance poles -with-the-unstable particles '- ef the-HSG-theøries. - - - - . - . - . - . - . - . - . -

\section{Acknowledgments}

This research is supported partially by CICYT (AEN99-0589), DGICYT (PB96-0960), and the EC Commission (TMR Grant FMRX-CT96-0012).

\section{References}

[1] Particle Data Group, Eur. Phys. J. C 3 (1998) 1.

[2] J.L. Miramontes and C.R. Fernández-Pousa, Phys. Lett. B 472 (2000) 392

[3] C.R. Fernández-Pousa, M.V. Gallas, T.J. Hollowood, and J.L. Miramontes, 'N $484(1997) 609$

[4] C.R. Fernández-Pousa, M.V. Gallas, T.J. Hollowood, and J.L. Miramontes, 'Nucl. Phys. B' $499(1997) 673$

[5] G. Breit and E.P. Wigner, IPhys. Rev. 49 519

R.J. Eden, P.V. Landshoff, D.I. Olive and J.C. Polkinghorne, The analytic S-matrix, Cambridge Univ. Press (1966);

S. Weinberg, The Quantum Theory of Fields (Vol. I), Cambridge Univ. Press (1995).

[6] C.R. Fernández-Pousa and J.L. Miramontes, 'Nucl. Phys. B $\mathbf{5 1 8}(1998)$ 745.

[7] O.A. Castro-Alvaredo, A. Fring, C. Korff, and J.L. Miramontes, ${ }^{i} \mathrm{Nucl}$. Phys. B $\mathbf{5} \mathbf{7} \mathbf{5}(2000) 535$.

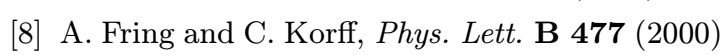
$380^{\prime}$

[9] O.A. Castro-Alvaredo, A. Fring and C. Korff, Phys. Lett. B $\mathbf{4 8 4}(2000) 16 \overline{7}_{\text {; }}$

O.A. Castro-Âlvaredo and A. Fring, Identifying the Operator Content, the Homogeneous SineGordon models, hep-th/0008044; Renormalization group flow with unstable particles, hepth/0008208.

[10] A.N. Kirillov, J. Soviet Math. 47 (1989) 2450; V.V. Bazhanov and N. Reshetikhin, J. Physics A 23 (1990) 1477;

A. Kuniba, 'Nucl. Phys. B $\mathbf{3 8 9}(1993)$ 209.

[11] Al.B. Zamolodchikov, Resonance Factorised Scattering and Roaming Trajectories, preprint ENS-LPS-335 (1991);

M.J. Martins, 'Phys. Rev. Lett. $\mathbf{6 9}(1992) 2461$; 'Nucl. Phys. B 394 (1993) 339; P. Dorey and F. Ravanini, Int. J. Mod. Phys. A 8 (1993) 873. 